ARTICLE

\title{
Multi-parameter photon-by-photon hidden Markov modeling
}

\author{
Paul David Harris (1] ${ }^{1 凶}$, Alessandra Narducci (10 ${ }^{2}$, Christian Gebhardt ${ }^{2}$, Thorben Cordes ${ }^{2}$, Shimon Weiss (1) ${ }^{3,4} \&$ \\ Eitan Lerner (1) ${ }^{1,5 凶}$
}

Single molecule Förster resonance energy transfer (smFRET) is a unique biophysical approach for studying conformational dynamics in biomacromolecules. Photon-by-photon hidden Markov modeling $\left(\mathrm{H}^{2} \mathrm{MM}\right)$ is an analysis tool that can quantify FRET dynamics of single biomolecules, even if they occur on the sub-millisecond timescale. However, dye photophysical transitions intertwined with FRET dynamics may cause artifacts. Here, we introduce multi-parameter $\mathrm{H}^{2} \mathrm{MM}\left(\mathrm{mpH}^{2} \mathrm{MM}\right)$, which assists in identifying FRET dynamics based on simultaneous observation of multiple experimentally-derived parameters. We show the importance of using $\mathrm{mpH}^{2} \mathrm{MM}$ to decouple FRET dynamics caused by conformational changes from photophysical transitions in confocal-based smFRET measurements of a DNA hairpin, the maltose binding protein, MalE, and the type-III secretion system effector, YopO, from Yersinia species, all exhibiting conformational dynamics ranging from the sub-second to microsecond timescales. Overall, we show that using $\mathrm{mpH}^{2} \mathrm{MM}$ facilitates the identification and quantification of biomolecular sub-populations and their origin.

\footnotetext{
${ }^{1}$ Department of Biological Chemistry, The Alexander Silberman Institute of Life Sciences, Faculty of Mathematics \& Science, The Edmond J. Safra Campus, The Hebrew University of Jerusalem, Jerusalem 9190401, Israel. ${ }^{2}$ Physical and Synthetic Biology. Faculty of Biology, Ludwig-Maximilians-Universität München, Großhadernerstr. 2-4, 82152 Planegg-Martinsried, Germany. ${ }^{3}$ Department of Chemistry and Biochemistry, and Department of Physiology, University of California, Los Angeles, CA, USA. ${ }^{4}$ CaliforniaNanoSystems Institute, University of California, Los Angeles, CA, USA. ${ }^{5}$ The Center for

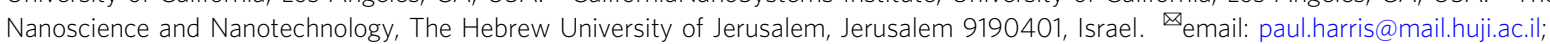
eitan.lerner@mail.huji.ac.il
} 
T he role of structural dynamics in biomolecular function has come to the forefront of biophysical research ${ }^{1,2}$. Biomolecules in solution exhibit structural dynamics at a hierarchy of timescales and modes, from bond rotations to movements of entire globular domains, occurring at times from picoseconds to seconds and longer ${ }^{3}$. In many cases, the stages in the biomolecular function are promoted by different sub-populations of closely-related structures, or conformations. Examples include coupling of catalytic activity to domain dynamics in some enzymes $^{4,5}$, the dynamics of the DNA bubble in transcription initiation to support transcription start site selection ${ }^{6,7}$, DNA mismatch repair ${ }^{8}$, protein translocation ${ }^{9}$, chaperone action ${ }^{10}$, the allosteric regulation of the AAA+ disaggregase ${ }^{11}$, active membrane transport ${ }^{12-17}$, and many other important biochemical processes, in which structural dynamics is coupled to or influences biological function ${ }^{1,2}$. Thus, methods capable of identifying and characterizing distinctly time-separated structural subpopulations of biomolecules are of great interest in biomolecular sciences and structural biology.

NMR- and EPR-based methods ${ }^{18-21}$ as well as single molecule methods ${ }^{22-26}$ have come to the forefront in the field of dynamic structural biology, each with their own advantages and limitations. Single molecule methods allow probing one biomolecule at a time while tracking multiple experimental parameters simultaneously. This approach provides access to conformational heterogeneity, real-time kinetics, and identification of rare conformational states otherwise masked due to ensemble averaging.

One of the most popular single molecule approaches relies on the phenomenon of Förster resonance energy transfer (FRET), single molecule FRET $\left(\right.$ smFRET) ${ }^{27}$, where the biomolecule of interest is site-specifically labeled at two strategic residues with two fluorescent dyes, which can exhibit transfer of excitation energy from the donor dye to the acceptor dye with a probability (or efficiency; $E$ ), which is inversely proportional to the sixth power of the distance between the dyes, according to the Förster relation ${ }^{28-30}$. The FRET efficiency can be determined either ratiometrically, through the donor and acceptor fluorescence intensities, or through the use of fluorescence lifetime-based methods. Ratiometric methods yield an initial raw efficiency, $E_{\text {raw }}$ (see Supplementary Eq. 1), to which correction factors must be applied, such as leakage of donor photons into the acceptor channel, direct excitation of the acceptor by the donor light source, differences in donor and acceptor fluorescence quantum yields and detection efficiencies (better known as the $\gamma$-factor), in order to yield accurate $E^{31-33}$. Lifetime-based approaches do not require such corrections, but rely on pulsed laser sources and timecorrelated single photon counting modules ${ }^{34}$. SmFRET has proven to be a powerful tool to disentangle conformational subpopulations of biomacromolecules undergoing dynamic transitions over a range of timescales ${ }^{3}$. Nevertheless, smFRET remains limited by the time resolution and observation time of the apparatus ${ }^{3}$. A popular approach is the observation of individual freely-diffusing molecules through the excitation volume of a confocal microscope $\mathrm{e}^{1,2}$. Here the observation time of a single molecule is on the order of a few milliseconds, with possible time resolution of dynamics as rapid as nanoseconds using advanced analyses of photon statistics within single molecule photon bursts (Fig. 1a, b). Some of the latter methods include photon distribution analysis, or probability distribution analysis (PDA $)^{35-40}$, burst variance analysis (BVA $)^{41}$, FRET two-kernel density estimator (FRET-2CDE) ${ }^{42}$, analysis of two-dimensional histograms of donor fluorescence lifetimes, and ratiometric FRET efficiencies of bursts, also known as FRET lines ${ }^{34,43,44}$, fluorescence correlation spectroscopy (FCS) 55,46 coupled to FRET ${ }^{47-49}$, maximum likelihood approaches $^{50-54}$, such as hidden Markov modeling $4,7,55,56$ (HMM), and photon recoloring 57,58 . These have been summarized in recent reviews of the field ${ }^{1,2}$.

Photon-by-photon hidden Markov modeling $\left(\mathrm{H}^{2} \mathrm{MM}\right)^{56}$ is a maximum likelihood method 57,59 that adopts the HMM machinery, while working directly with the photon data without binning into fluorescence intensity time traces, other than the clock time of the acquisition card, e.g. $50 \mathrm{~ns}$ for nsALEX, $12.5 \mathrm{~ns}$

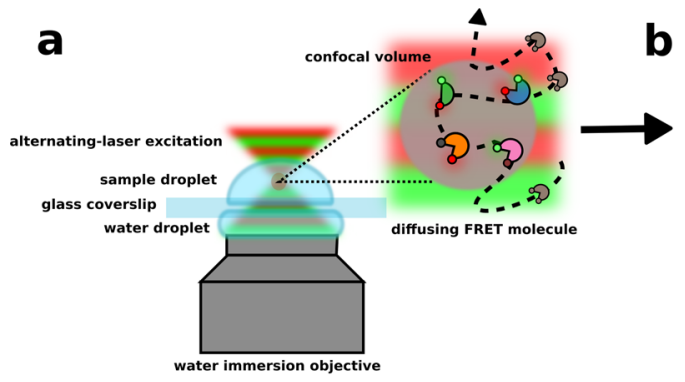

b
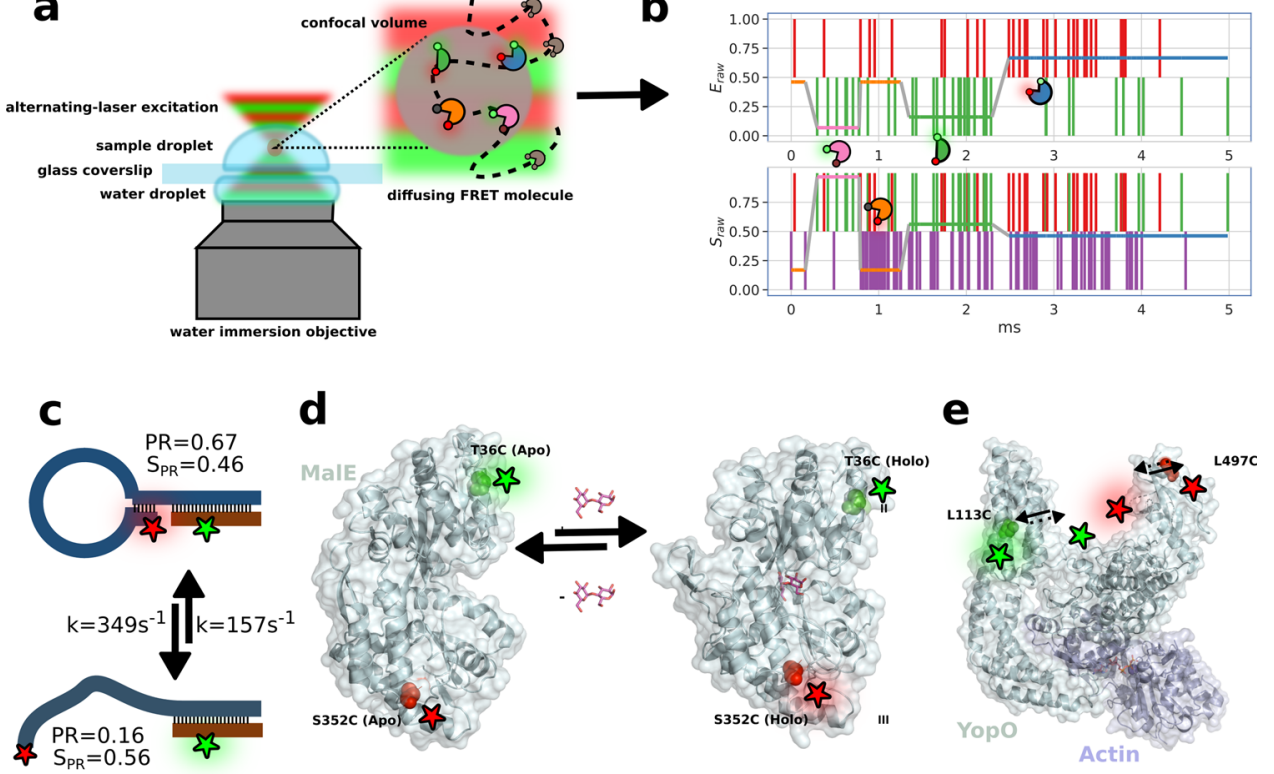

Fig. 1 Cartoon representations of data acquisition, and biological systems examined in this work. a Confocal microscope setup with inset illustrating the diffusive trajectory of a single molecule in and out of the confocal volume, undergoing conformational and photophysical changes, producing, $\mathbf{b}$ a photon time trace; photons represented by vertical bars, and the most likely state path according to the Viterbi algorithm overlayed as horizontal colored line. c-e Biological systems studied: c DNA hairpin, $\mathbf{d}$ maltose binding protein MalE with structure-guided conformational changes, and e type III secretion effector YopO. See Supplementary Fig. 1 for a version of this figure with transition rates, $E_{\text {raw }}$ and $S_{\text {raw }}$ values included for the biological systems. 
for $\mu$ sALEX. $\mathrm{H}^{2} \mathrm{MM}$ can extract the number of states involved in the underlying FRET dynamics, their mean $E_{\text {raw }}$ values and transition rate constants. Nevertheless, while advanced smFRET setups often detect multiple fluorescence parameters beyond the intensities, such as in alternating laser excitation (ALEX) ${ }^{60,61}$ or in multi-color smFRET-based measurements ${ }^{62-69}, \mathrm{H}^{2} \mathrm{MM}$ in its current iteration only uses the raw FRET efficiency of a single donor-acceptor pair of dyes.

Here, we introduce multi-parameter $\mathrm{H}^{2} \mathrm{MM}\left(\mathrm{mpH}^{2} \mathrm{MM}\right)$, which enables incorporation of multiple parameters in the analysis, through additional photon streams. We demonstrate this concept with two types of ALEX experiments: microsecond ALEX ( $\mu$ sALEX) and nanosecond ALEX (nsALEX; known also as pulsed interleaved excitation, PIE ${ }^{60,61}$. We applied this approach to different biomacromolecular complexes with dynamics ranging from the sub-second to microsecond timescales: (i) a DNA hairpin loop ${ }^{70}$, (ii) the maltose binding protein MalE from E. coli, and (iii) YopO, a type-III-secretion system effector from pathogenic Yersinia species ${ }^{71}$ (Fig. 1c-e, Supplementary Fig. 1). Our results and analysis demonstrate that $\mathrm{mpH}^{2} \mathrm{MM}$ is able to quantitatively report sub-populations based on both the ALEXrelevant mean parameters, $E_{\text {raw }}$ and the stoichiometry, $S_{\text {raw }}$ (see Supplementary Eq. 2), as well as their transition rate constants, demonstrating FRET-relevant conformational transitions, as well as FRET-irrelevant photophysical transitions. We also present the H2MM_C python package ${ }^{72}$, with a backend written in C, for data processing, which is approximately two orders of magnitude faster than the previous implementation of $\mathrm{H}^{2} \mathrm{MM}$ in matlab ${ }^{56}$.

Importantly, throughout this work, we make the clear distinction between sub-populations and states, where the latter is referred to the state models used to describe the dynamically interconverting sub-populations resolved from the data. This distinction is important, since thermodynamic states are single potential wells, and it is possible that the identified subpopulations are actually a group of states that interconvert much faster than the time resolution of the measurements. It should also be noted that we use the term parameter in multiparameter $\mathrm{H}^{2} \mathrm{MM}$ to refer to parameters derived from ratios of sums of photons in different photon steams (e.g., E and S). These are distinct from state model parameters (e.g., rate constants, mean E).

\section{Results}

Verification of $\mathrm{mpH}^{2} \mathrm{MM}$ against simulated data. Analysis with single parameter $\mathrm{H}^{2} \mathrm{MM}\left(\mathrm{spH}^{2} \mathrm{MM}\right)$ and $\mathrm{mpH}^{2} \mathrm{MM}$ can be performed using any given state model. Therefore, we must select the most likely state model among several, differing in their number of states and number of transition rate constants. Discriminating over- and under-fitted state models from the most likely model has proven difficult in the past ${ }^{7,73}$. Previously, we proposed the modified Bayes information criterion (BIC'), which does not provide an extremum-based decision on the most likely state model $^{7}$. In the current work, we implement the integrated complete likelihood (ICL $)^{74,75}$, which gets a minimum value for the most likely state model, as the primary criterion for state model selection.

Using simulated smFRET data, where the ground truth of the number and properties of the states is known, we find that the ICL is more reliable than the BIC' at selecting the most reliable state model (see Supplementary Fig. 2, and Jupyter notebooks in supplementary dataset ${ }^{72}$ ). Yet, there are instances in the simulated data, and in real data sets, we describe later, where the selection of the most likely state model based on ICL is of a model with too few states, relative to our prior knowledge of the system. Therefore, we always consider the ICL first, then BIC', and take into account the prior knowledge of the system when selecting the most likely state model (see Supplementary Note 2 for expanded discussion, Supplementary Fig. 2).

To verify the validity of the multi-parameter approach, we perform a series of simple simulations (see supplementary Jupyter notebook mpH2MMsimulations $\mathrm{s}^{76}$ ). We compare results of $\mathrm{spH}^{2} \mathrm{MM}$ and $\mathrm{mpH}^{2} \mathrm{MM}$ analyses of simulated data where the acceptor excitation photon stream was either included or excluded. Using this data, we find that selecting the most likely state model based on the ICL parameter reliably identifies the correct ground truth state model, and this model accurately reproduces the transition rate constants, $E_{\text {raw }}$ and $S_{\text {raw }}$ values used in the simulation (Supplementary Figs. 3 and $4, E_{\text {raw }}$, Supplementary Table 1, $S_{\text {raw }}$ values defined in Supplementary Eqs. 6 and 7, respectively). In contrast, $\mathrm{spH}^{2} \mathrm{MM}$ is less reliable, and depending on the circumstances, it is unable to distinguish states with similar $E_{\text {raw }}$ values, which are easily distinguished in $\mathrm{mpH}^{2} \mathrm{MM}$ by their $S_{\text {raw }}$ values. Further, without the information about $S_{\text {raw }}$, interpretation of the models is more difficult, even if the correct number of states and their accurate $E_{\text {raw }}$ values are recovered in $\mathrm{spH}^{2} \mathrm{MM}$.

DNA hairpin exhibiting millisecond dynamics. As a first biological test system for $\mathrm{mpH}^{2} \mathrm{MM}$, we used a DNA hairpin system introduced by Tsukanov et al. with a loop containing 31 adenines and a six base-pair stem ${ }^{70}$. The opening and closing rate constants of the hairpin vary as a function of the GC content of the stem as well as the sodium chloride $(\mathrm{NaCl})$ concentration ${ }^{70}$. When appropriately labeled with a FRET donor and acceptor pair of dyes (ATTO 550 and ATTO $647 \mathrm{~N}$, respectively), the open and closed hairpin sub-populations exhibit distinct low and high mean $E_{\text {raw }}$ values, respectively. The hairpin containing two GCs out of the six stem bases, which we term HP3, exhibited opening and closing rates of a few milliseconds, depending on the $\mathrm{NaCl}$ concentration in the buffer. Such a DNA construct with wellcharacterized and tunable transition rates serves as an ideal model system to test and characterize the performance of $\mathrm{mpH}^{2} \mathrm{MM}$.

We first perform nsALEX measurements ${ }^{61}$ with this construct at a concentration of $300 \mathrm{mM} \mathrm{NaCl}$, where a mix of both open and closed states are expected to interchange dynamically ${ }^{70}$. As a qualitative test for FRET dynamics occurring within bursts, we use burst variance analysis $(\mathrm{BVA})^{41}$, which compares the expected variance in $E_{\text {raw }}$ based on shot noise (the static FRET semi-circle) against the actual variance in $E_{\text {raw }}$. BVA of the HP3 data shows clear deviation from the static FRET semi-circle, suggesting that individual HP3 molecules are undergoing FRET dynamics as they traverse the confocal volume, which we term within-burst FRET dynamics (Fig. 2a). E- $\tau_{\mathrm{D}}$ plots $^{44}$ also indicate within-burst dynamics (see Supplementary Fig. 5). However, without the prior knowledge of the DNA hairpin behavior as a two-state FRET system, and without knowing how many more sub-populations unrelated to FRET may exist, it is not necessarily clear how many distinct sub-populations are involved in withinburst dynamics. In visual examination of the $2 \mathrm{D}$ E-S plot, three sub-populations are apparent: (i) an open hairpin sub-population with mean $E_{\text {raw }}$ of 0.2 , (ii) a closed hairpin sub-population, with a $E_{\text {raw }}$ of 0.65 , both open and closed sub-populations have mean $S_{\text {raw }}$ of 0.5 , and (iii) a third sub-population with a mean $E_{\text {raw }}$ of 0 , and mean $S_{\text {raw }}$ of 1 , where the acceptor is either in a dark state, or missing altogether (Fig. 2b). The 2D E-S plot also exhibits bursts with intermediate $E_{\text {raw }}$ values, bridging between the open and closed hairpin sub-populations. As these bursts are particularly dynamic in the BVA analysis, these are bursts where the hairpin is undergoing opening and closing transitions while crossing the confocal volume. 


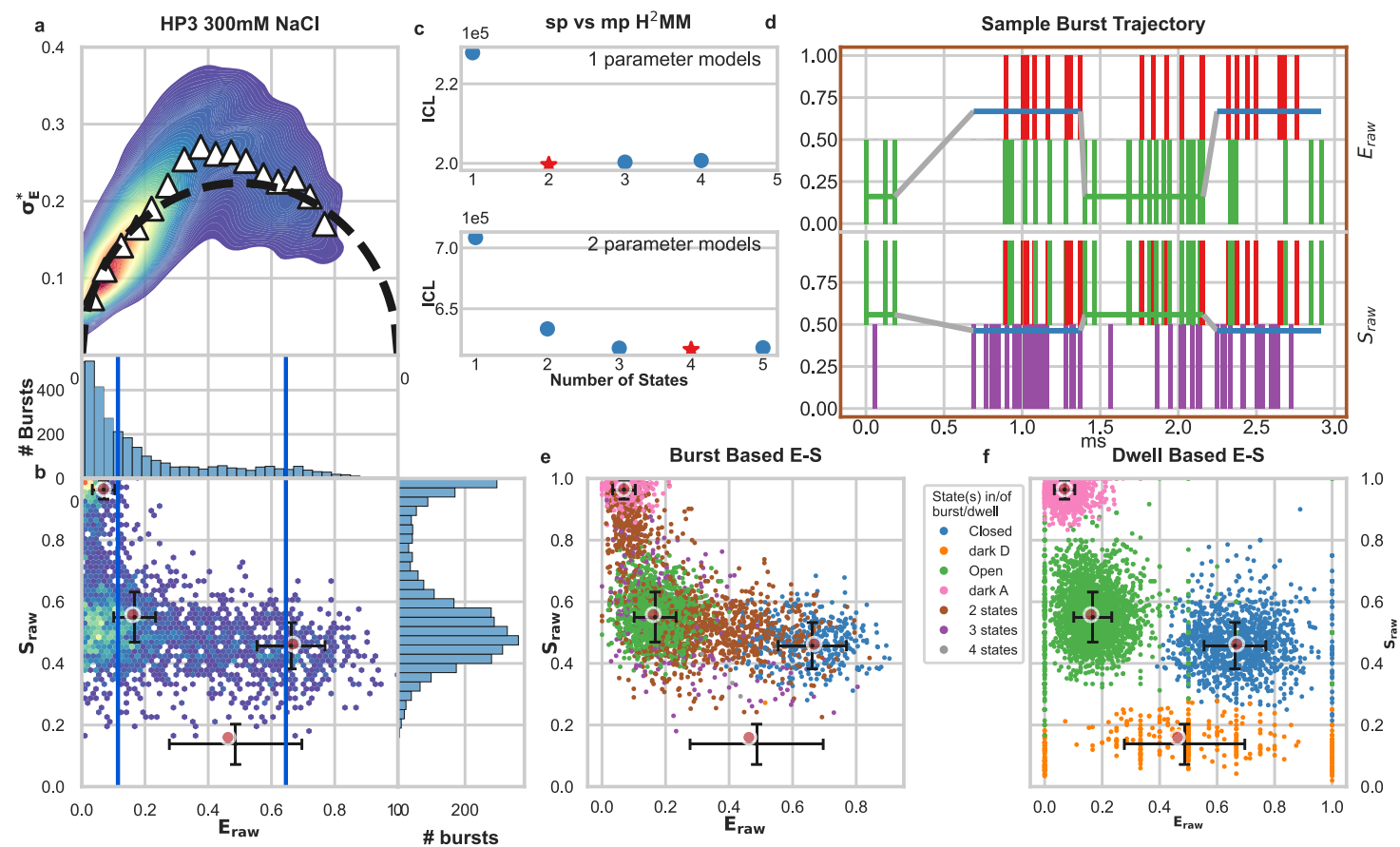

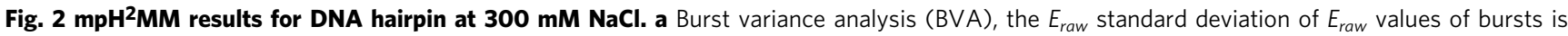
displayed versus their $E_{\text {raw }}$ values. Bursts with $E_{\text {raw }}$ standard deviations higher than expected solely from shot noise (semi-circle), indicate dynamic heterogeneity, such as within-burst FRET dynamics. Triangles indicate the average of standard deviation values per $E_{\text {raw }}$ bin. $\mathbf{b} 2 \mathrm{D}$ histogram of $E_{\text {raw }}$ and $S_{\text {raw }}$ (E-S plots, colloquially) of bursts. The $E_{\text {raw }}$ and $S_{\text {raw }}$ values of sub-populations derived from $\mathrm{mpH}^{2} \mathrm{MM}$ are marked by red circles, and the $E_{\text {raw }}$ and $S_{\text {raw }}$ standard deviation of these values, derived from the Viterbi dwell time analysis, are marked by black crosses. c Comparison of values of the integrated complete likelihood (ICL) of $\mathrm{spH}^{2} \mathrm{MM}$ (top panel) and $\mathrm{mpH}^{2} \mathrm{MM}$ (bottom panel) of optimized models with different state models. The most likely state model is marked as a red star. $\mathbf{d}$ A sample burst trajectory, with photons represented as colored vertical bars, with donor excitation photons colored green and red for donor and acceptor, respectively. Acceptor excitation photons are colored purple. $E_{\text {raw }}$ (top panel) and $S_{\text {raw }}$ (bottom panel) of sub-populations determined from dwells using the Viterbi algorithm, are overlayed on the photon bars, and colored to indicate the state of the dwell. The border color also represents the type of burst. e, $\mathbf{f} \mathrm{E}-\mathrm{S}$ scatter plots of data processed by the Viterbi algorithm. Consecutive photons with the same state are considered as a single dwell, $E_{\text {raw }}$ and $\mathrm{S}_{\text {raw }}$ values are then calculated as in Supplementary Eqs. 8 and 9, respectively. $\mathrm{MpH}^{2} \mathrm{MM}$-derived sub-populations and Viterbi-derived $E_{\text {raw }}$ and $S_{\text {raw }}$ standard deviations (SD) are overlayed as red circles and black crosses, respectively. e, $\mathbf{f}$ E-S scatter plot of bursts e or dwells within bursts $\mathbf{f}$, color coded by which states are present in the bursts (e) or according to the state of the dwell (dwell based $E_{\text {raw }}$ and $S_{\text {raw }}$ defined in Supplementary Eqs. 8 and 9, respectively) $\mathbf{f}$, according to Viterbi algorithm. Color coding is the same throughout $\mathbf{d}, \mathbf{e}$, and $\mathbf{f}$. Error bars (s.d.) in $\mathbf{b}$, e, and $\mathbf{f}$ are the same, with $n=1232,466,3033$, and 1493 dwells for the closed, dark donor, open, and dark acceptor states, respectively. See E- $\tau_{\mathrm{D}}$ analysis in Supplementary Fig. 5, and more examples of bursts classified by the Viterbi algorithm in Supplementary Fig. 6.

Analyses of this data with $\mathrm{spH}^{2} \mathrm{MM}$ and $\mathrm{mpH}^{2} \mathrm{MM}$ show different patterns in the ICL values of the state models. The ICL is minimized for $\mathrm{spH}^{2} \mathrm{MM}$ models for a two-state model, while it is minimized for a four-state model when using $\mathrm{mpH}^{2} \mathrm{MM}$. Visual inspection of the one-dimensional projection of burst data onto the $E_{\text {raw }}$ parameter immediately suggests an explanation for this discrepancy, as it appears as only two sub-populations. The donor-only or dark acceptor state, and the open hairpin state exhibit similar low $E_{\text {raw }}$ values and are difficult to distinguish as sub-populations based solely on $E_{\text {raw }}$. This projection reflects the data accessible to $\mathrm{spH}^{2} \mathrm{MM}$, the donor excitation streams, and thus the open hairpin and dark acceptor states are expected to have nearly identical FRET signatures with regard to the streams accessible to $\mathrm{spH}^{2} \mathrm{MM}$, thus leading to the false inference of only two states. The open hairpin FRET sub-population and the dark acceptor states are, however, quite distinct with regard to the acceptor excitation stream, which is accessible to $\mathrm{mpH}^{2} \mathrm{MM}$.

In the ICL-based selected four-state model retrieved by $\mathrm{mpH}^{2} \mathrm{MM}$, two out of the four states match nicely with the states in the ICL-based selected model from $\mathrm{spH}^{2} \mathrm{MM}$ model, having similar $E_{\text {raw }}$ values. Their $S_{\text {raw }}$ values are $\sim 0.5$ (Fig. $2 \mathrm{~b}$ red circles), as expected for molecules undergoing FRET. The third and fourth states in the model can be matched to dark acceptor and dark donor sub-populations, respectively. The third state has a $E_{\text {raw }}$ value $\sim 0$ and a $S_{\text {raw }}$ value $\sim 1$ (Fig. 2b, top left red circle). This state has a clear sub-population of bursts associated with it in the 2D E-S plot. The fourth state has an intermediate $E_{\text {raw }}$ value, and a very low $S_{\text {raw }}$ value of $\sim 0.17$ (Fig. 2b, bottom red circle, Supplementary Table 2). There is no obvious sub-population visually observed in the E-S plots to which this would correspond, but the $E_{\text {raw }}$ and $S_{\text {raw }}$ values are consistent with this being a dark donor state. More importantly, comparing the parameters of the state models retrieved by $\mathrm{spH}^{2} \mathrm{MM}$ and $\mathrm{mpH}^{2} \mathrm{MM}$, we find that the transition rate constants derived using $\mathrm{mpH}^{2} \mathrm{MM}$ are closer to those found by Tsukanov et al. ${ }^{70}$ than those extracted using $\mathrm{spH}^{2} \mathrm{MM}$ (Supplementary Table 3, and supplementary .csv files of all state models found by $\mathrm{H}^{2} \mathrm{MM}$ analysis ${ }^{72}$. The transition rate constants provide a clue as to why the fourth state does not show up in the E-S plots as a distinct subpopulation, as the transition rates predict rare transitions to it, and rapid transitions away from it. Thus, populating the fourth state occurs only briefly and rarely in bursts undergoing rapid dynamics, such that it does not appear as a clear sub-population in the E-S plots (Supplementary Table 3, and supplementary .csv file ${ }^{72}$ ).

The Viterbi algorithm finds the most likely state path through each burst, given a state model and its parameter values (Fig. $2 \mathrm{~d}$ and Supplementary Fig. 6a-e). We use this to classify bursts by which states are present within each burst (Fig. 2d, Supplementary Fig. 6f), and separate photons into dwells, for which $E_{\text {raw }}$ and 
$S_{\text {raw }}$ can be defined (Fig. 2d, Supplementary Eqs. 8 and 9 in Supplementary Note 1.3). Additional analysis of dwells and their durations is provided in Supplementary Fig. 7. Visual examination of the burst-based E-S plot (Fig. 2e) shows that the Viterbi algorithm reasonably classifies most bursts that have $E_{\text {raw }}$ and $S_{\text {raw }}$ values close to the predicted value of a given sub-population as only having that state present, as well as bursts with intermediate $E_{\text {raw }}$ and $S_{\text {raw }}$ that are predicted to include dwells of multiple states. Notably, there are only a few bursts classified as having dwells solely in the dark donor state (Fig. 2e), keeping with what is predicted by the transition rates, and indeed, few dwells are even found in this state (Fig. 2f, Supplementary Fig. 6g). The scarcity of the donor dark state in the Viterbi analysis serves to both confirm this observation and prove the sensitivity of $\mathrm{mpH}^{2} \mathrm{MM}$ at the same time. In summary, using $\mathrm{spH}^{2} \mathrm{MM}$, we do not properly decouple the FRET-relevant information from the FRET-irrelevant dye transitions to fluorophore dark states for the DNA hairpin data, which influences the accuracy of the retrieved values for the $E_{\text {raw }}$ and rate constant parameters. On the other hand, using $\mathrm{mpH}^{2} \mathrm{MM}$ assists in the proper decoupling of the FRET-relevant information from the FRET-irrelevant ones and in gaining accurate parameter values. See Supplementary Figs. 8-19 for additional hairpin data acquired at different concentrations of $\mathrm{NaCl}$. Now that we have verified $\mathrm{mpH}^{2} \mathrm{MM}$ with a well-defined biomolecular system of the DNA hairpin, HP3, we move to explore its usefulness in other biomacromolecular systems.

Quantifying the dynamics of a substrate-binding protein. In the previous example, we examined a system that exhibits intrinsic conformational dynamics, hence dynamics that is not induced by binding of a ligand. Now, we test $\mathrm{mpH}^{2} \mathrm{MM}$ on a system with conformational dynamics that is induced by substrate binding. For this, we select the periplasmic maltose binding protein, MalE from E. coli ${ }^{77}$, which is the extracellular component of the maltose $\mathrm{ABC}$ importer $\mathrm{MalFGK}_{2}-\mathrm{E}^{77}$. MalE is a bilobed protein with a structural core built from a periplasmic binding protein (PBP)-like II domain. Two rigid domains, $\mathrm{D}_{1}$ and $\mathrm{D}_{2}$, are separated by a two-segment $\beta$-strand hinge and are complemented by a C-terminal embellishment that facilitates structural dynamics between open and closed states ${ }^{17}$. This allows for MalE to close upon substrate binding, similar to a venus flytrap. For our nsALEX smFRET measurements we produced a MalE double-cysteine variant with labels at the outer sides of the two lobes, specifically residues T36C and S352C. As shown previously, this enables tracking of the opening and closing dynamics in single MalE molecules ${ }^{17,78}$. We test three concentrations of the substrate maltose: none (apo), $1 \mu \mathrm{M}$ (close to the $K_{D}$ value $^{77}$ ) and $1 \mathrm{mM}$ (holo). FRET histograms, using a dual channel burst search $(\mathrm{DCBS})^{36}$ filter exhibit three sub-populations: (i) a minor, low $E_{\text {raw }}$ sub-population at $E_{\text {raw }}$ of 0.1 , (ii) a major sub-population with an intermediate $E_{\text {raw }}$ of 0.5 , and (iii) a major sub-population with a high $E_{\text {raw }}$ of 0.7 . We use DCBS because the donor- and acceptor-only sub-populations are very strong, and otherwise overwhelm the nsALEX data. Since we apply DCBS, bursts of the high $S_{\text {raw }}$ and low $E_{\text {raw }}$ values cannot represent molecules with permanently dark acceptor, but could be the result of either a real conformation, or of frequent acceptor blinking. With increasing maltose concentration, the fraction of the $\sim 0.5 E_{\text {raw }}$ subpopulation decreases, while the fraction of the $\sim 0.7 E_{\text {raw }}$ subpopulation increases (Fig. 3).

The BVA plot exhibits evidence of within-burst dynamics, so $\mathrm{mpH}^{2} \mathrm{MM}$ analysis of within-burst dynamics is warranted (Fig. 3, top row).

In $\mathrm{mpH}^{2} \mathrm{MM}$ analysis, the ICL-based model selection identifies the five-state model for $1 \mu \mathrm{M}$ maltose, and the four-state model for $1 \mathrm{mM}$ maltose. Examining these models, we find that all contain a single high $S_{\text {raw }}$ state and a single low $S_{\text {raw }}$ state, with the high $S_{\text {raw }}$ state also having an $E_{\text {raw }}$ of 0 , and importantly, no bursts exist in these ranges due to the use of a DCBS filter (Supplementary Tables 4 and 5). Therefore, we can conclude that these states are the result of transition in the donor and acceptor dyes for the low and high $S_{\text {raw }}$ states, respectively. The transition rates of the models and Viterbi analysis both show that these states are appreciably populated (Fig. 3, bottom row, Supplementary Figs. 20-22, Supplementary Table 7, and supplementary .csv file ${ }^{72}$ ), thus the use of $\mathrm{mpH}^{2} \mathrm{MM}$ analysis is vital here. Depending on the maltose concentration, the ICL of $\mathrm{spH}^{2} \mathrm{MM}$ analysis predicts different numbers of states for each concentration, and the $E_{\text {raw }}$ values of the states within these models are far less consistent (Fig. 3, vertical bars). These states are often similar to states found by $\mathrm{mpH}^{2} \mathrm{MM}$, but their interpretation would be ambiguous if we did not have $\mathrm{mpH}^{2} \mathrm{MM}$ for additional information. In other cases, $\mathrm{spH}^{2} \mathrm{MM}$-based states appear as a fusion of two states found by $\mathrm{mpH}^{2} \mathrm{MM}$.

As another example of how vital $\mathrm{mpH}^{2} \mathrm{MM}$ analysis is in this case, consider the BVA signature of the apo form. Our analysis shows that the FRET dynamics for $E_{\text {raw }}$ is not due to the actual dynamics between the open conformations of MalE. This is clear since the $\sim 0.7 E_{\text {raw }}$ sub-population is not identified if maltose is not supplied. Such interpretation cannot be made from $\mathrm{spH}^{2} \mathrm{MM}$ results, due to the less consistent prediction of the number of states, and the parameters of those models. Therefore, we can confirm that MalE undergoes large-scale conformational dynamics linked to its function, mostly induced by the binding of maltose, hence it follows an induced-fit binding mechanism.

Adapting to $\mu$ sALEX: microsecond dynamics of YopO. Finally, we demonstrate how to apply $\mathrm{mpH}^{2} \mathrm{MM}$ with $\mu$ sALEX experiments. For this, we use the type-III secretion effector from Yersinia species, $\mathrm{YopO}^{71}$. We measure the conformational dynamics of a double-cysteine variant of YopO, with dyes labeling residues L113C and L497C. These labeling positions are expected to change distances upon binding to actin. Burst selection is performed using the DCBS filter, for the same reasons as in the MalE data - there are strong blinking dynamics that overwhelm the analysis otherwise. Interestingly, in the absence of actin there appears to be a single FRET sub-population in E-S plots, with tails towards dark donor and dark acceptor sub-populations. Nevertheless, BVA shows these bursts have a variance above the expected static FRET semi-circle (Fig. 4a, top panel), and hence within-burst dynamics. In the presence of bound actin $(60 \mu \mathrm{M})$, a main sub-population is present with a shift toward lower $E_{\text {raw }}$ values, and the BVA plot suggests no signature of within-burst dynamics at that main sub-population (Fig. 4b, top panel).

Using this $\mu$ sALEX data with $\mathrm{mpH}^{2} \mathrm{MM}$, the alternation period proves to be an obstacle, causing $\mathrm{mpH}^{2} \mathrm{MM}$ analysis to fail without a key adjustment to the data. Unlike in nsALEX, multiple photons can be detected during a given alternation period of the donor or acceptor excitation lasers. This results in photons originating from donor excitation that are temporally separated from photons originating from acceptor excitation in a periodic pattern, resulting in alternating periods where no photons originating from donor excitation are detected, and alternatively periods where no photons originating from acceptor excitation are detected. When we first apply $\mathrm{mpH}^{2} \mathrm{MM}$ to $\mu$ sALEX data, we find that instead of detecting states with meaningful $S_{\text {raw }}$ values, all states have $S_{\text {raw }}$ values of either 0 or 1 , and transition rates are all very similar to the alternation rate, meaning that $\mathrm{mpH}^{2} \mathrm{MM}$ detects the alternation rate instead of actual conformational dynamics (Supplementary Fig. 23a, b). 

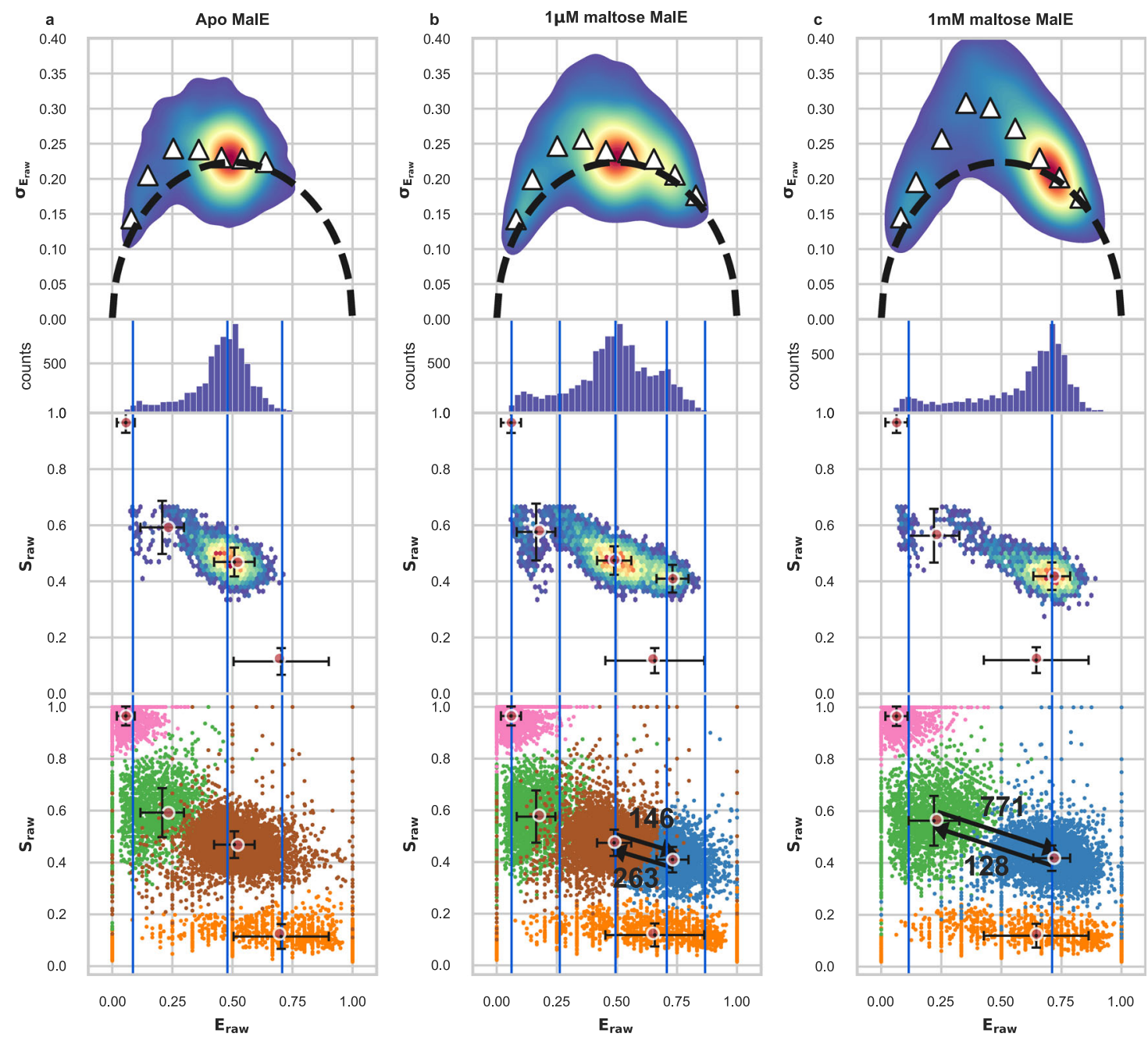

Fig. 3 Results for MalE. Top row: BVA of concatenated dataset. Upper middle row: $E_{\text {raw }}$ histogram of bursts. Lower middle row: E-S plot of bursts, with ICLbased selected results overlayed, red circles indicating the values derived from the ICL-based selected $\mathrm{mpH}^{2} \mathrm{MM}$ state model, and the black crosses the standard deviation of the Viterbi-derived dwell $E_{\text {raw }}$ and $S_{\text {raw }}$ values. Vertical blue lines represent the $E_{\text {raw }}$ values of the states from the ICL-based selected $\mathrm{spH}^{2} \mathrm{MM}$ state model. Bottom row: Dwell based E-S plots as in Fig. 2, with transition rates (in units of $\mathrm{s}^{-1}$ ) between selected states indicated by arrows added. a apo MalE, b $1 \mu \mathrm{M}$ maltose, c $1 \mathrm{~m} \mathrm{M}$ maltose. Error bars (s.d.) for a: $n=1556,3925,3042,8665$ dwells for dark donor, dark acceptor, low FRET, and mid FRET states, respectively, for $\mathbf{b} n=2505,7753,4793,9083,4010$ dwells for dark donor, dark acceptor, low FRET, mid FRET, and high states FRET, respectively, for (c) $n=1586,5539,4196,7550$ dwells for dark donor, dark acceptor, low FRET, and high FRET states, respectively.

In that respect, to enable meaningful $\mu$ sALEX analysis via $\mathrm{mpH}^{2} \mathrm{MM}$ that incorporates photons originating from acceptor excitation, we introduce a shift so that the times of the acceptor excitation photons overlap with the photons originating from donor excitation (see Supplementary Note 1.1.1). By doing so, the alternation period is no longer detected and meaningful dynamics with $E_{\text {raw }}$ and $S_{\text {raw }}$ values can be recovered (Fig. 4, Supplementary Fig. 23c). The usefulness of this analysis is evidenced by the detection of dark donor and dark acceptor states. Thus application of $\mathrm{mpH}^{2} \mathrm{MM}$ even to $\mu$ sALEX data usually yields better results than with $\mathrm{spH}^{2} \mathrm{MM}$. However, caution must be taken to avoid artefacts due to the alternation period. For instance, if the timescale of a transition approaches that of the alternation period, $S_{\text {raw }}$ values may be biased or averaged together due to the shift (for in-depth discussion on this topic, see Supplementary Note 1.1.2).

Applying $\mathrm{mpH}^{2} \mathrm{MM}$ to analyze the measured data of YopO in the presence of actin, the most likely model is clearly a four-state model, using an alternation period of $50 \mu \mathrm{s}(20 \mathrm{kHz}$ alternation rate). The ICL-based model selection identifies four states, while the BIC'-based selection shows the four-state model to be close to the 0.005 threshold, and the five-state model can be further disregarded based on its reasonableness. Selection is more difficult for the apo results, as the two criteria disagree with ICL-based model selection that identifies three states and BIC'-based model selection that identifies five. Therefore, the most likely model is either the three-, four-, or five-state model, and examination of these models and prior knowledge of the data is necessary. The three-state model predicts states that appear as dark donor and acceptor, and a single FRET state. This model can be ruled out because the BVA shows significant dynamics around the single FRET population, and thus the single FRET state is insufficient to explain the BVA signature. The five-state model, on the other hand, suffers from the opposite problem - there are two states with very low $S_{\text {raw }}$ values, where it appears as though the dark donor state has split into two. The four-state model, however, is reasonable, showing two FRET states, dark donor and dark acceptor states (Supplementary Tables 6 and 7). Transition rates between the high and low FRET states are 12,400 $\mathrm{s}^{-1}$ and 6,000 $\mathrm{s}^{-1}$ for transitions from high $E_{\text {raw }}$ to low $E_{\text {raw }}$ states, and for transitions from low $E_{\text {raw }}$ to high $E_{\text {raw }}$ states, respectively. These dynamics, 

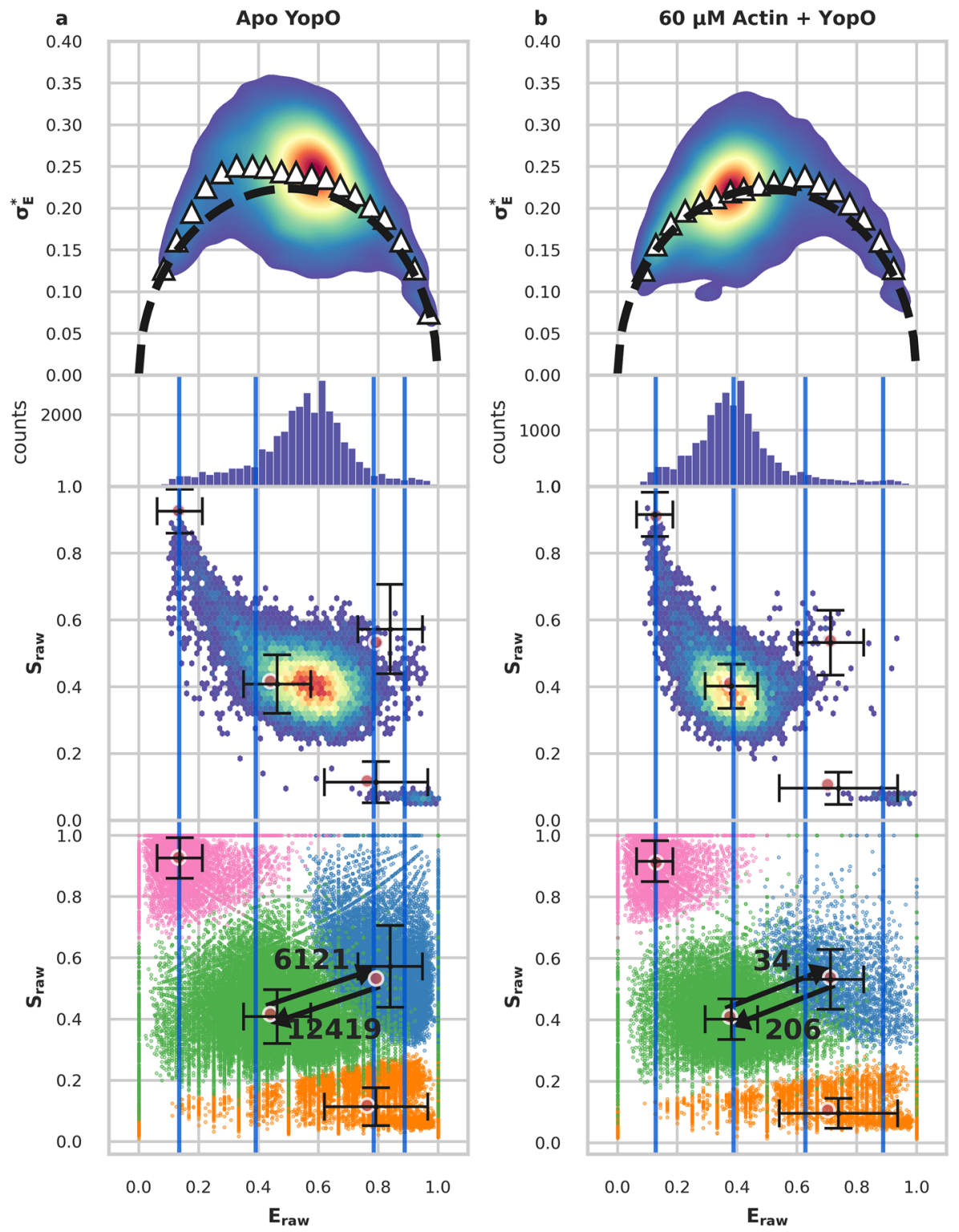

Fig. 4 Results for YopO. a, b Top row: BVA analysis. Upper middle row: $E_{\text {raw }}$ histogram of bursts, lower middle row: E-S plot of bursts. Red dots indicate $\mathrm{mpH}^{2} \mathrm{MM}$ states. Bottom row: Dwell based E-S plots as in Fig. 2, with transition rates (in units of $\mathrm{s}^{-1}$ ) between selected states indicated by arrows added. Vertical bars indicate the $E_{\text {raw }}$ values of the states for the $\mathrm{BIC}^{\prime}$-based $\mathrm{spH}_{\text {Eraw }}$ model. a apo YopO exhibiting sub-millisecond dynamics. b YopO with $60 \mu \mathrm{M}$ actin exhibiting slower within-burst dynamics, and a shift toward the lower FRET conformation. Error bars (s.d.) for a $n=40,723,89,613,108,641$, and 89,613 dwells for dark donor, dark acceptor, low FRET, and high FRET states respectively, for $\mathbf{b} n=11,518,9279,31,100$, and 3105 dwells for dark donor, dark acceptor, low FRET, and high FRET states, respectively.

however, approach the timescale of the alternation rate $(20 \mathrm{kHz}$; for detailed discussion and examination, see Supplementary Note 1.1.2 as well as Supplementary Figs. 24, 25). Based on the analyses of the Viterbi-derived dwell times, error analysis of data sub-samples, and comparison with the results of $\mathrm{mpH}^{2} \mathrm{MM}$ analysis employed on other measurements using different alternation periods, we conclude that these transition rates are not artifacts, and reflect true FRET transitions in the data (see Supplementary Note 1.1.2, Supplementary Figs. 26-28 for comparison of different alternation periods, Supplementary Tables 8 and 9 for optimized model of different alternation period).

The timescale of the FRET dynamics being faster than burst duration by two orders of magnitude explains the appearance of the data in the FRET histogram as a single FRET population, yet with a signature of within-burst dynamics in the BVA plot. Inspecting the results of the $\mathrm{mpH}^{2} \mathrm{MM}$ analysis, the meaning of the within-burst FRET dynamics of YopO in the absence of actin becomes clear - it exhibits transitions in the tens of microseconds between two main FRET states intertwined with rapid transitions to dark donor and dark acceptor states. Each burst that lasts a few milliseconds contains multiple dwells in the underlying states and transitions between them, and so the bursts are averaged-out as a single main population. When comparing these results, with the analysis results of YopO in the presence of bound actin, it becomes clear that the lower $E_{\text {raw }}$ state of the two FRET states in the absence of actin is stabilized upon actin binding. Therefore, we can conclude that YopO conformational dynamics relevant to actin binding occurs intrinsically, regardless of the presence of actin, and that actin stabilizes and locks one of the pre-existing conformations.

Without using $\mathrm{mpH}^{2} \mathrm{MM}$, it would have been difficult to accurately report on this dynamics, as the FRET within-burst 
dynamics is intertwined with FRET-irrelevant transitions to dark states. It should be noted that we have successfully decoupled conformational and photophysical dynamics in $\mu$ sALEX data without the use of fluorescence lifetimes.

\section{Discussion}

$\mathrm{MpH}^{2} \mathrm{MM}$ increases both the information content of the results and the sensitivity of the $\mathrm{H}^{2} \mathrm{MM}$ algorithm to differences in the photon streams that are too subtle when examining only a single parameter. We have shown that $\mathrm{mpH}^{2} \mathrm{MM}$ is able to disentangle dark acceptor states from low FRET states that have structural meaning. We have exhibited the advantage of using $\mathrm{mpH}^{2} \mathrm{MM}$ to elucidate an accurate quantitative picture on two proteins with two types of conformational dynamics that serve their function: (1) MalE with conformational dynamics induced by maltose binding, and (2) YopO with conformational dynamics occurring intrinsically, with actin binding stabilizing one of the states. In both cases, the overall picture is complicated by having the FRETrelevant transitions intertwined with the FRET-irrelevant dye transition to dark states, and not taking these into account could result in wrongly elucidated quantities and potentially wrong interpretations. Of note is the rapid conformational dynamics on the order of tens of microseconds in YopO when actin was absent. The exact description of the dynamics was possible using $\mathrm{mpH}^{2} \mathrm{MM}$ on $\mu$ sLLEX, and hence did not necessarily require analysis of the correlation of donor fluorescence lifetimes with ratiometric FRET values, as can be done using FRET lines fits to E- $\tau_{\mathrm{D}} 2 \mathrm{D}$ plots in lifetime-based smFRET ${ }^{44}$. As $\mu$ sALEX and nsALEX setups are now commonly used, the acceptor excitation stream is usually available, therefore, $\mathrm{mpH}^{2} \mathrm{MM}$ maximizes the use of available data for characterizing rapidly interconverting sub-populations.

$\mathrm{MpH}^{2} \mathrm{MM}$ is, therefore, a powerful tool for the quantification of rapid conformational dynamics in a variety of systems, while also extracting information that can be used to extract inter-dye distance distributions. The integration of the acceptor excitation photon stream is critical in this process, as we have shown that $\mathrm{spH}^{2} \mathrm{MM}$ often conflates photophysical and conformational states, leading to incorrect $E_{\text {raw }}$ and transition rate constants. Comparing a given protein or other biomacromolecular system with different ligands, or concentrations of ligands, it is possible to discriminate when a system demonstrates intrinsic conformational dynamics or conformational changes triggered by ligands. $\mathrm{MpH}^{2} \mathrm{MM}$ provides accurate quantitative measures of both transition rates and mean $E_{\text {raw }}$ values, the latter of which can be converted into accurate mean FRET efficiency values with the proper correction factors for the system ${ }^{79}$. Such information can then be converted into mean inter-dye distances, which provide invaluable information for FRET-based integrative structural models ${ }^{7,33,79}$.

The success of integrating the acceptor excitation stream into our analysis, suggests that a similar approach could also be employed in camera-based smFRET applications. Alternating laser excitation, and HMM algorithms are commonly employed in analysis of data, although the information of the acceptor excitation stream is discarded in these analyses, and only used for truncating trajectories upon acceptor bleaching. The present $\mathrm{mpH}^{2} \mathrm{MM}$ algorithm is inappropriate for such data as the information is based on intensity and a constant frame rate, instead of single photon arrivals with variable interphoton times. Alternatively, the introduction of SPAD arrays ${ }^{80}$ should allow analysis of immobilized molecules with single photon precision, which would allow for analysis of such data directly using $\mathrm{mpH}^{2} \mathrm{MM}$.

$\mathrm{mpH}^{2} \mathrm{MM}$ is also not restricted to our demonstrated application in two detector setups with nsALEX and $\mu$ sALEX. The most obvious application of $\mathrm{mpH}^{2} \mathrm{MM}$ beyond ALEX, is with the multiple photon streams in multi-parameter fluorescence detection (MFD) $)^{34,43}$, or with multi-color smFRET-based measurements ${ }^{62-69}$. Here, three or even four spectrally-distinct dyes are attached to the biomolecule of interest, and each produces a distinct photon stream. This enables the simultaneous observation of multiple inter-dye FRET efficiencies at once. If qualitative tests indicate that such a system is undergoing withinburst dynamics, $\mathrm{mpH}^{2} \mathrm{MM}$ is well-suited to extract the transfer efficiencies relevant to the underlying dynamically interconverting sub-populations. Applying these methods is as simple as assigning an index to each photon stream. We include a supplementary Jupyter notebook using a developer version of FRETBursts ${ }^{81}$ that accepts fluorescence anisotropy information from multi-parameter fluorescence detection, or from MFD coupled to pulsed interleaved excitation ${ }^{34,43}$, and demonstrate $\mathrm{mpH}^{2} \mathrm{MM}$ 's ability to disentangle fluorescence anisotropies on data kindly provided by Cao et al. ${ }^{82}$. Values within the emission probability matrix can then be used as intensities to calculate all relevant ratiometric values. Multiple conformational subpopulations interconverting at sub-millisecond timescales could be simultaneously measured and disentangled with such a setup. Information on fluorescence anisotropy could also be incorporated, which, depending on the labeling scheme could report on dye steric restriction or oligomeric state of the system in question.

In this work, we used two ratiometric parameters drawn from ratios of photon counts of the photon streams available in ALEXbased measurements within the $\mathrm{mpH}^{2} \mathrm{MM}$ framework. In some smFRET measurements, such as in nsALEX, the photon nanotimes, which are the basis for fluorescence lifetime data, can also be considered as a parameter within the $\mathrm{mpH}^{2} \mathrm{MM}$ framework. However, unlike $E_{\text {raw }}$ and $S_{\text {raw }}$, which are approximately binomially distributed, photon nanotimes distribute exponentially or sometimes according to a sum of exponentials. To transform photon nanotime data into a parameter that is also centrally distributed, and hence one that can be used within the $\mathrm{mpH}^{2} \mathrm{MM}$ framework, we propose a method for mapping the nonbinomially distributed lifetime to a binomially distributed parameter amenable to $\mathrm{mpH}^{2} \mathrm{MM}$ (see Supplementary Note 3 for further details).

The new H2MM_C python package makes $\mathrm{H}^{2} \mathrm{MM}$ analysis much more practical, most analysis, for up to six states, take less time than the data acquisition times, given our modest hardware (a 2 year old middle-tier gaming laptop). See Supplementary Note 4 and Supplementary Tables 10 and 11 for system requirements and the duration of calculations in this paper. The supplied Jupyter notebooks provide examples for how to execute $\mathrm{mpH}^{2} \mathrm{MM}$ using FRETBursts. Experimenters using other platforms must utilize their knowledge of the fine details of their data to properly filter and cast their data into the simple and general format that the H2MM_C package ${ }^{76}$ accepts. We also provide an in-depth tutorial available on Zenodo ${ }^{83}$.

\section{Methods}

Production of YopO and MalE variants. The double-cysteine variant YopO L113C/L497C is produced and purified as described in Peter et al. ${ }^{71}$ and kindly provided by Gregor Hagelüken and Martin Peter, Institute of Structural Biology (University of Bonn). The double-cysteine variant MalE T36C/S352C is generated and purified according to methods reported previously ${ }^{17}$

Labeling of MalE. The MalE variant T36C/352C is stochastically-labeled with Alexa Flour $^{\text {Ts }} 555$ and Alexa Fluor ${ }^{\text {tw }} 647$ dye derivatives as described in Peter et al. and deBoer et al. ${ }^{17,84}$. The His ${ }_{6}$-MalE double variant $(200 \mu \mathrm{g})$ is incubated with $1 \mathrm{mM}$ DTT and loaded immediately after on $200 \mu \mathrm{L}$ (wet volume) Ni-Sepharose 6 Fast Flow resin, pre-equilibrated with labeling buffer $1(50 \mathrm{mM}$ Tris- $\mathrm{HCl} \mathrm{pH} 7.4$, $50 \mathrm{mM} \mathrm{KCl}$ ). After a washing step with 50 column volumes labeling buffer 1 , the loaded resin is incubated overnight at $4{ }^{\circ} \mathrm{C}$ with 5 -fold excess $(25 \mathrm{nmol}$ of each fluorophore dissolved in $1 \mathrm{~mL}$ of labeling buffer 1 . Next, the resin is further washed 
with 50 column volumes labeling buffer 1 to remove the excess unbound fluorophores. Labeled protein is eluted with $800 \mu \mathrm{L}$ elution buffer $(50 \mathrm{mM}$ Tris- $\mathrm{HCl} \mathrm{pH}$ $8.0,50 \mathrm{mM} \mathrm{KCl}, 500 \mathrm{mM}$ imidazole) and further purified by size-exclusion chromatography (ÄKTA pure system, Superdex 75 Increase 10/300 GL column, GE Healthcare). Protein concentration is determined using the protein extinction coefficient and corrected for direct absorption of the fluorophores at $280 \mathrm{~nm}$. Labeling efficiencies are estimated to be at least $60 \%$ for each fluorophore individually and donor-acceptor pairing at least $20 \%$.

Labeled MalE is stored in $50 \mathrm{mM}$ Tris- $\mathrm{HCl} \mathrm{pH} 7.4,50 \mathrm{mM} \mathrm{KCl}$ and $1 \mathrm{mgmL}^{-1}$ bovine serum albumin (BSA) at $4{ }^{\circ} \mathrm{C}$ for no more than 3 days. Concentrations ranged between 10 to $100 \mathrm{nM}$.

Labeling of YopO. The protein variant YopO L113C/L497C is stochasticallylabeled with fluorophore-linked maleimide derivatives, as described previously ${ }^{84}$ Briefly, $200 \mu \mathrm{g}$ of protein is incubated with $5 \mathrm{mM}$ DTT at $4{ }^{\circ} \mathrm{C}$ for $30 \mathrm{~min}$, to prevent oxidation of the cysteine thiol groups. The protein is loaded onto a PD Mini-Trap G-25 column (GE Healthcare) pre-equilibrated with Buffer A (50 mM Tris- $\mathrm{HCl} \mathrm{pH} \mathrm{7.4,50} \mathrm{mM} \mathrm{KCl)} \mathrm{and} \mathrm{subsequently} \mathrm{eluted} \mathrm{with} 1 \mathrm{~mL}$ of Buffer A by gravity gel filtration, in order to eliminate the excess of DTT. The eluted protein is incubated overnight at $4{ }^{\circ} \mathrm{C}$ with $50 \mathrm{nmol}$, respectively, of Alexa Fluor ${ }^{\text {mox }} 555$ - and Alexa Fluor ${ }^{\mathrm{mt}}$ 647- $\mathrm{C}_{2}$ maleimide (ThermoFisher Scientific). Excess dyes are removed again by gravity gel filtration using a PD Min-Trap G-25 column, as described above. The labeled protein is further purified from residual dyes and soluble aggregates by size-exclusion chromatography (SEC), with a Superdex ${ }^{\text {tix }} 75$ Increase 10/300 GL column, on an ÄKTA pure system (GE Healthcare). Protein concentration is determined using the protein extinction coefficient and corrected for direct absorption of the fluorophores at $280 \mathrm{~nm}$.

Labeling efficiencies are estimated to be at least $60 \%$ for each fluorophore individually and donor-acceptor pairing at least $20 \%$.

\section{Experimental setup}

Experimental setup for studies of HP3. We performed the nsALEX smFRET measurements of the doubly-labeled DNA hairpin construct ${ }^{70}$ in the presence of 50, $100,200,250,300$, and $350 \mathrm{mM}$ sodium chloride, using a confocal-based setup (ISS $^{\mathrm{TM}}$, USA) assembled on top of an Olympus IX73 inverted microscope stand. We use a pulsed picosecond fiber laser $(\lambda=532 \mathrm{~nm}$, pulse width of 100 ps FWHM, operating at $20 \mathrm{MHz}$ repetition rate and $100 \mu \mathrm{W}$ measured at the back aperture of the objective lens) for exciting the Cy3B donor dye (FL-532-PICO, CNI, China), and a pulsed picosecond diode laser $(\lambda=642 \mathrm{~nm}$, pulse width of $100 \mathrm{ps}$ FWHM, operating at $20 \mathrm{MHz}$ repetition rate and $60 \mu \mathrm{W}$ measured at the back aperture of the objective lens) for exciting the ATTO 647N acceptor dye (QuixX $642-140$ PS, Omicron, $\mathrm{GmbH}$ ), delayed by $25 \mathrm{~ns}$. The laser beams pass through a polarization maintaining optical fiber and then further shaped by a linear polarizer and a halfwave plate. A dichroic beam splitter with high reflectivity at 532 and $640 \mathrm{~nm}$ (ZT532/640rpc, Chroma, USA) reflects the light through the optical path to a high numerical aperture $(\mathrm{NA})$ super apochromatic objective $(60 \mathrm{X}, \mathrm{NA}=1.2$, water immersion, Olympus, Japan), which focuses the light onto a small confocal volume. The microscope collects the fluorescence from the excited molecules through the same objective, and focuses it with an achromatic lens $(\mathrm{f}=100 \mathrm{~mm}$ ) onto a $100 \mu \mathrm{m}$ diameter pinhole (variable pinhole, motorized, tunable from $20 \mu \mathrm{m}$ to $1 \mathrm{~mm}$ ), and then re-collimates it with an achromatic lens $(\mathrm{f}=100 \mathrm{~mm})$. Then, donor and acceptor fluorescence are split between two detection channels using a dichroic mirror with a cutoff wavelength at $\lambda=652 \mathrm{~nm}$ (FF652-Di01-25x36, Semrock Rochester NY, USA). We further filter the donor and acceptor fluorescence from other light sources 585/40 nm (FF01-585/40-25, Semrock Rochester NY, USA) and 698/70 nm (FF01-698/70-25, Semrock Rochester NY, USA) band-pass filters, respectively, and detect the donor and acceptor fluorescence signals using two hybrid photomultipliers (Model R10467U-40, Hamamatsu, Japan), routed through a 4-to-1 router to a time-correlated single photon counting (TCSPC) module (SPC150 , Becker \& Hickl, GmbH) as its START signal (the STOP signal is routed from the laser controller). We perform data acquisition using the VistaVision software (version 4.2.095, 64-bit, ISS ${ }^{\mathrm{TM}}$, USA) in the time-tagged time-resolved (TTTR) file format. After acquiring the data, we transform it into the photon HDF5 file format ${ }^{85}$ for easy dissemination of raw data to the public, and easy input in the FRETBursts analysis software.

Experimental setup for studies of MalE. The nsALEX measurements on MalE are performed using a home-built setup, assembled around an Olympus IX73 inverted microscope stand. We use a picosecond pulsed diode laser $(\lambda=532 \mathrm{~nm}$, pulse width of 100 ps FWHM, operating at $20 \mathrm{MHz}$ repetition rate and $32 \mu \mathrm{W}$ at the back aperture of the objective) for exciting the Alexa Fluor ${ }^{\text {min }} 555$ donor (LDH-P-FA$530 \mathrm{~B}$, Picoquant $\mathrm{GmbH})$, and a picosecond pulsed diode laser $(\lambda=640 \mathrm{~nm}$, pulse width of 90 ps FWHM, operating at $20 \mathrm{MHz}$ repetition rate, and $20 \mu \mathrm{W}$ at the back aperture of the objective) to excite the Alexa Fluor ${ }^{\text {mI }} 647$ acceptor (LDH-D-C-640, Picoquant, GmbH), driven by the same PDL828 "Sepia II" (Picoquant, GmbH) controller. The laser light is guided into the microscope by a dual-edge beamsplitter (ZT532/640rpc Chroma/AHF, GmbH) and focused to a diffraction-limited excitation spot by an oil immersion objective (UPLSAPO 60XO, Olypus). The emitted light is collected through the same objective, spatially filtered through a $50 \mu \mathrm{m}$ pinhole, and spectrally split into donor and acceptor channels by a single-edge dichroic mirror (H643 LPXR, AHF). The emission is filtered (donor: BrightLine HC 582/75, Semrock/AHF, acceptor: Longpass 647 LP Edge Basic, Semrock/AHF) and the signal is recorded with avalanche photodiodes (SPCM-AQRH-34, Excelitas) and a TCSPC module (HydraHarp400, Picoquant, GmbH). Data was acquired with Picoquant SymPhoTime 64 v2.7.

Coverslips are passivated with $1 \mathrm{mg} \mathrm{mL}^{-1}$ BSA in PBS buffer before adding around $100 \mu \mathrm{L}$ of sample. MalE stock solution is diluted to $\sim 50 \mathrm{pM}$ concentration in $50 \mathrm{mM}$ Tris- $\mathrm{HCl} \mathrm{pH} \mathrm{7.4,50} \mathrm{mM} \mathrm{KCl}$, and either, none, $1 \mu \mathrm{M}$ or $1 \mathrm{mM}$ of the ligand maltose.

Experimental setup for studies of YopO. The $\mu$ sALEX measurements of YopO are performed using the setup in Gebhardt et al. ${ }^{86}$. These are conducted on the same home-built microscope as the MalE experiments, built around an Olympus IX71 base, although the lasers and dichroics are replaced as described below. We use a continuous wave $\lambda=532 \mathrm{~nm}$ diode laser (OBIS 532-10-LS, Coherent, USA) laser with $60 \mu \mathrm{W}$ power measured at the back aperture of the objective to excite the donor Alexa Fluor ${ }^{\mathrm{m}} 555$ dye, and a continuous wave $\lambda=640 \mathrm{~nm}$ diode laser (OBIS 640-100-LX, Coherent, USA) with $25 \mu \mathrm{W}$ power measured at the back aperture of the objective. The lasers are distally modulated by TTL pulses with an alternating frequency of $10 \mathrm{kHz}, 20 \mathrm{kHz}$, and $100 \mathrm{kHz}$, for an alternation period of $100 \mu \mathrm{s}$ $50 \mu \mathrm{s}$, and $10 \mu \mathrm{s}$, respectively. The lasers are combined and coupled into a polarization maintaining single-mode patch cable (P-3-488PM-FC2, Thorelabs, USA). The laser light is reflected into the objective by a dual-edge dichroic mirror (ZT532/ 640rpc, Chroma/AHF) and focused by a water immersion objective (UPlanSApo $60 / 1.2 \mathrm{w}$, Olympus, $\mathrm{GmbH}$ ). The dichroic mirrors, fluorescent filters and avalanche photodiodes are identical to those used for acquisiton of MalE data.

Coverlips are passivated with BSA as in MalE measurements. $100 \mu \mathrm{L}$ of YopO solution, diluted to between $50 \mathrm{pM}$ and $80 \mathrm{pM}$ is used for each measurement in $50 \mathrm{mM}$ Tris- $\mathrm{HCl} \mathrm{pH} \mathrm{7.4,50} \mathrm{mM} \mathrm{KCl}$. For measurements with actin, the buffer also contained $50 \mu \mathrm{M}$ non-muscle human actin protein (Cytoskeleton, Inc) and $0.2 \mathrm{mM}$ ATP and $0.2 \mathrm{mM} \mathrm{CaCl}_{2}$.

Data is acquired using labVIEW v7.1 software as presented in Ingargiola et al. ${ }^{87}$.

Burst selection. All data processing and analysis is performed using Jupyter Notebooks available in supplementary dataset, along with the accompanying photon-HDF5 files containing the raw data ${ }^{72}$. We perform burst search and selection using the FRETBursts analysis software ${ }^{88}$. The background is assessed per each $30 \mathrm{~s}$ of acquisition, and bursts are identified as time periods were the instantaneous photon count rate of a sliding window of $m=10$ consecutive photons is at least $F=6$ times higher than the background rate. Bursts in the normal selection are selected if they include at least 30 photons in total between all streams. Visualizations are performed using FRETBursts' dplot function, or matplotlib when greater customization is desired.

Single and multi-parameter $\mathbf{H}^{\mathbf{2}} \mathbf{M M}$ analysis. Bursts identified by FRETBursts are then converted into a format readable by the H2MM_C software ${ }^{76}$, by a simple function supplied in the Jupyter notebooks available in supplementary dataset ${ }^{72}$, this function is also responsible for applying the shift to acceptor excitation photons in $\mu$ sALEX experiments (Supplementary Note 1.1.1). In $\mathrm{spH}^{2} \mathrm{MM}$, only photons arising from donor excitation are considered, assigned to either donor or acceptor streams, identified by index 0 or 1 , respectively, depending on at which detector they arrived. $\mathrm{MpH}^{2} \mathrm{MM}$ also considers photons arriving during acceptor excitation, assigning these photons an index of $2 . \mathrm{All}^{2} \mathrm{MM}$ calculations are performed within the Jupyter notebooks, available in supplementary dataset ${ }^{72}$, using the Python package by Paul David Harris ${ }^{76}$. We use the $\mathrm{H}^{2} \mathrm{MM}$ algorithm (both single- and multi-parameter) to test how well different state models describe the data.

Model selection. To choose the best model, we primarily use the ICL ${ }^{74,75}$, where the state model reaching a minimal ICL is generally considered the one that describes the data best, with minimal free parameters. We always calculate sufficient numbers of state models to ensure ICL is minimized. The ICL parameter is defined in Eq. (1):

$$
\operatorname{ICL}(m)=-2 \ln \left(\mathbf{p}\left(\mathbf{y}, \hat{\mathbf{s}} \mid m, \hat{\lambda_{m}}\right)\right)+K \ln (n)
$$

where $\ln \left(\mathbf{p}\left(\mathbf{y}, \hat{\mathbf{s}} \mid m, \hat{\lambda_{m}}\right)\right)$ is the posterior probability of the most likely state path, as determined by the Viterbi algorithm, $K$ is the number of free parameters in the model, and $n$ is the number of photons in all bursts in the data set. $K$ is calculated as in Eq. (2):

$$
K=q^{2}+(r-1) q-1
$$

where $q$ is the number of states the state model represents, and $r$ is the number of photon streams used for the calculation of all of the parameters that are assessed. For $\mathrm{spH}^{2} \mathrm{MM}, r=2$, while for nsALEX $\mathrm{mpH}^{2} \mathrm{MM}, r=3$. The ICL is preferable as an extremum-based criterion over the previously proposed threshold based on the modified Bayes Information Criterion (BIC') ${ }^{7}$. See supplementary dataset ${ }^{72}$ for Jupyter notebooks testing the reliability of ICL with simulated data sets generated using PyBroMo ${ }^{89}$ (https://github.com/OpenSMFS/PyBroMo/releases/tag/0.8.1; was 
utilized in previous works ${ }^{7,85,90}$ ). We use the Viterbi algorithm to find the most likely state path based on the posterior probability.

Viterbi analysis. From the state path, photons are separated into dwells, each of which can be assigned a duration, a mean $E_{\text {raw }}$, and for $\mathrm{mpH}^{2} \mathrm{MM}$, a mean $S_{\text {raw }}$. This also allows bursts to be classified by which and how many states are present. As one measure of error, we use the weighted standard deviation and the weighted standard error of the $E_{\text {raw }}$ and $S_{\text {raw }}$ as a proxy for the standard error of the $\mathrm{H}^{2} \mathrm{MM}$ model (see Supplementary Note 1.3 for full derivation).

Error analysis by variance of subsets. Analysis of the variance of subsets is another method to assess the error of parameters (see Supplementary Note 1.4 for detailed description). This is implemented as a function in the Jupyter notebooks in the supplementary dataset ${ }^{72}$. This is an attractive approach, as it does not depend on any most likely state path like in the Vieterbi based approach. This method, however, is significantly more computationally expensive than the Viterbi approach.

Reporting summary. Further information on research design is available in the Nature Research Reporting Summary linked to this article.

\section{Data availability}

The photon-HDF5 data, Jupyter notebooks, .csv data and H2MM_C code that support the findings of this study are available in the Zenodo and github repositories with the identifiers at: https://doi.org/10.5281/zenodo.5566809, and https://doi.org/10.5281/ zenodo. $5535302^{72,76}$.

\section{Code availability}

The H2MM_C library used in this study is available on github https://github.com/ harripd/H2MMpythonlib (commit 1f3d0a84f149d21a740161372526eb3742027602). The FRETbursts used in this study is available on github https://github.com/harripd/ FRETBursts (commit 315c60d3791aa93cf2ec6e880003174c8192fc88). The phconvert code used $\mathrm{n}$ this study is available on github https://github.com/Photon-HDF5/phconvert v0.9 (commit 3a86e58f11f77e21c2a02ald9453060db681lc9c). The PyBroMo code used in this study is available on github: https://github.com/tritemio/PyBroMo v0.8.1 (commit 8403ae750ff68796ef4118dd497478cf54355382). labVIEW code is available on github: https://github.com/multispot-software/MultichannelTimestamper.

Received: 15 April 2021; Accepted: 3 February 2022; Published online: 22 February 2022

\section{References}

1. Lerner, E. et al. Toward dynamic structural biology: Two decades of singlemolecule Förster resonance energy transfer. Science 359, eaan1133 (2018).

2. Lerner, E. et al. FRET-based dynamic structural biology: Challenges, perspectives and an appeal for open-science practices. eLife 10, e60416 (2021).

3. Schuler, B. \& Hofmann, H. Single-molecule spectroscopy of protein folding dynamics-expanding scope and timescales. Curr. Opin. Struct. Biol. 23, 36-47 (2013).

4. Aviram, H. Y. et al. Direct observation of ultrafast large-scale dynamics of an enzyme under turnover conditions. Proc. Natl. Acad. Sci. 115, 3243-3248 (2018).

5. Mazal, H. \& Haran, G. Single-molecule FRET methods to study the dynamics of proteins at work. Curr. Opin. Biomed. Eng. 12, 8-17 (2019).

6. Robb, N. C. et al. The transcription bubble of the RNA polymerase - promoter open complex exhibits conformational heterogeneity and millisecond-scale dynamics: implications for transcription start-site selection. J. Mol. Biol. 425, 875-885 (2013).

7. Lerner, E., Ingargiola, A. \& Weiss, S. Characterizing highly dynamic conformational states: The transcription bubble in RNAP-promoter open complex as an example. J. Chem. Phys. 148, 123315 (2018).

8. Cristóvão, M. et al. Single-molecule multiparameter fluorescence spectroscopy reveals directional MutS binding to mismatched bases in DNA. Nucleic Acids Res. 40, 5448-5464 (2012).

9. Fessl, T. et al. Dynamic action of the Sec machinery during initiation, protein translocation and termination. eLIFE 7, e35112 (2018).

10. Calabrese, A. N. et al. Inter-domain dynamics in the chaperone SurA and multi-site binding to its outer membrane protein clients. Nat. Commun. 11, 2155 (2020).

11. Mazal, H. et al. Tunable microsecond dynamics of an allosteric switch regulate the activity of a AAA+ disaggregation machine. Nat. Commun. 10, 1438 (2019).

12. Zhao, Y. et al. Single-molecule dynamics of gating in a neurotransmitter transporter homologue. Nature 465, 188-193 (2010).
13. Zhao, Y. et al. Substrate-modulated gating dynamics in a Na+-coupled neurotransmitter transporter homologue. Nature 474, 109-113 (2011).

14. Erkens, G. B., Hänelt, I., Goudsmits, J. M. H., Slotboom, D. J. \& van Oijen, A. M. Unsynchronised subunit motion in single trimeric sodium-coupled aspartate transporters. Nature 502, 119-123 (2013).

15. Gouridis, G. et al. Conformational dynamics in substrate-binding domains influences transport in the ABC importer GlnPQ. Nat. Struct. Mol. Biol. 22, 57-64 (2015).

16. Husada, F. et al. Conformational dynamics of the $\mathrm{ABC}$ transporter $\mathrm{McjD}$ seen by single-molecule FRET. EMBO J. 37, 1-13 (2018).

17. de Boer, M. et al. Conformational and dynamic plasticity in substrate-binding proteins underlies selective transport in ABC importers. eLIFE 8, e44652 (2019).

18. Anthis, N. J. \& Clore, G. M. Visualizing transient dark states by NMR spectroscopy. Q. Rev. Biophysics. 48, 35-116 (2015).

19. Clore, G. M. \& Iwahara, J. Theory, practice, and applications of paramagnetic relaxation enhancement for the characterization of transient low-population states of biological macromolecules and their complexes. Chem. Rev. 109, 4108-4139 (2009).

20. Palmer, A. G. NMR characterization of the dynamics of biomacromolecules. Chem. Rev. 104, 3623-3640 (2004).

21. Ravera, E. et al. Insights into domain-domain motions in proteins and RNA from solution NMR. Acc. Chem. Res. 47, 3118-3126 (2014).

22. Su, Q. P. \& Ju, L. A. Biophysical nanotools for single-molecule dynamics. Biophysical Rev. 10, 1349-1357 (2018).

23. Bavishi, K. \& Hatzakis, N. Shedding light on protein folding, structural and functional dynamics by single molecule studies. Molecules 19, 19407-19434 (2014).

24. Medina, E., R. Latham, D. \& Sanabria, H. Unraveling protein's structural dynamics: from configurational dynamics to ensemble switching guides functional mesoscale assemblies. Curr. Opin. Struct. Biol. 66, 129-138 (2021).

25. Mandal, S. S. Force spectroscopy on single molecules of life. ACS Omega. 5, 11271-11278 (2020)

26. Dimura, M. et al. Quantitative FRET studies and integrative modeling unravel the structure and dynamics of biomolecular systems. Curr. Opin. Struct. Biol. 40, 163-185 (2016).

27. Ha, T. et al. Probing the interaction between two single molecules: fluorescence resonance energy transfer between a single donor and a single acceptor. Proc. Natl Acad. Sci. 93, 6264-6268 (1996).

28. Förster, T. Zwischenmolekulare energiewanderung und fluoreszenz. Ann. der Phys. 437, 55-75 (1948).

29. Förster, T. 10th spiers memorial lecture. transfer mechanisms of electronic excitation. Discuss. Faraday Soc. 27, 7 (1959).

30. Stryer, L. \& Haugland, R. P. Energy transfer: a spectroscopic ruler. Proc. Natl Acad. Sci. 58, 719-726 (1967)

31. Dahan, M. et al. Ratiometric measurement and identification of single diffusing molecules. Chem. Phys. 247, 85-106 (1999).

32. Deniz, A. A. et al. Single-pair fluorescence resonance energy transfer on freely diffusing molecules: Observation of Forster distance dependence and subpopulations. Proc. Natl Acad. Sci. 96, 3670-3675 (1999).

33. Lee, N. K. et al. Accurate FRET measurements within single diffusing biomolecules using alternating-laser excitation. Biophysical J. 88, 2939-2953 (2005).

34. Rothwell, P. J. et al. Multiparameter single-molecule fluorescence spectroscopy reveals heterogeneity of HIV-1 reverse transcriptase:primer/template complexes. Proc. Natl Acad. Sci. 100, 1655-1660 (2003).

35. Aviram, M., Felekyan, S., Gaiduk, A. \& Seidel, C. A. Separating structural heterogeneities from stochastic variations in fluorescence resonance energy transfer distributions via photon distribution analysis. J. Phys. Chem. B 110 6970-6978 (2006).

36. Nir, E. et al. Shot-noise limited single-molecule FRET histograms: comparison between theory and experiments. J. Phys. Chem. B 110, 22103-22124 (2006).

37. Kalinin, S., Felekyan, S., Antonik, M. \& Seidel, C. A. Probability distribution analysis of single-molecule fluorescence anisotropy and resonance energy transfer. J. Phys. Chem. B 111, 10253-10262 (2007).

38. Kalinin, S., Felekyan, S., Valeri, A. \& Seidel, C. A. Characterizing multiple molecular states in single-molecule multiparameter fluorescence detection by probability distribution analysis. J. Phys. Chem. B 112, 8361-8374 (2008).

39. Kalinin, S., Valeri, A., Antonik, M., Felekyan, S. \& Seidel, C. A. Detection of structural dynamics by FRET: a photon distribution and fluorescence lifetime analysis of systems with multiple states. J. Phys. Chem. B 114, 7983-7995 (2010).

40. Santoso, Y., Torella, J. P. \& Kapanidis, A. N. Characterizing single-molecule FRET dynamics with probability distribution analysis. ChemPhysChem 11, 2209-2219. (2010).

41. Torella, J. P., Holden, S. J., Santoso, Y., Hohlbein, J. \& Kapanidis, A. N. Identifying molecular dynamics in single-molecule fret experiments with burst variance analysis. Biophysical J. 100, 1568-1577 (2011). 
42. Tomov, T. E. et al. Disentangling subpopulations in single-molecule FRET and ALEX experiments with photon distribution analysis. Biophysical J. 102, 1163-1173 (2012).

43. Sisamakis, E., Valeri, A., Kalinin, S., Rothwell, P. J. \& Seidel, C. A. Accurate single-molecule FRET studies using multiparameter fluorescence detection. In Methods in Enzymology, vol. 475, 455-514 (Elsevier Inc., 2010), 1 edn. https:// doi.org/10.1016/S0076-6879(10)75018-7https://linkinghub.elsevier.com/ retrieve/pii/S0076687910750187.

44. Barth, A. et al. Unraveling multi-state molecular dynamics in single-molecule FRET experiments- Part I: Theory of FRET-Lines (2021). http://arxiv.org/abs/ 2107.14770.

45. Magde, D., Elson, E. \& Webb, W. Thermodynamic fluctuations in a reacting system-measurement by fluorescence correlation spectroscopy. Phys. Rev. Lett. 29, 705 (1972).

46. Rigler, R.et al. Fluorescence correlation spectroscopy with high count rate and low background: analysis of translational diffusion. In: Accounts of Chemical Research 22.10 (3 Oct. 1993), pp. 169-175. https://doi.org/10.1007/ BF00185777

47. Widengren, J., Schweinberger, E., Berger, S. \& Seidel, C. A. Two new concepts to measure fluorescence resonance energy transfer via fluorescence correlation spectroscopy: theory and experimental realizations. J. Phys. Chem. A 105, 6851-6866 (2001).

48. Torres, T. \& Levitus, M. Measuring conformational dynamics: a new FCSFRET approach. J. Phys. Chem. B 111, 7392-7400 (2007).

49. Gurunathan, K. \& Levitus, M. FRET fluctuation spectroscopy of diffusing biopolymers: contributions of conformational dynamics and translational diffusion. J. Phys. Chem. B 114, 980-986 (2010).

50. Köllner, M. \& Wolfrum, J. How many photons are necessary for fluorescencelifetime measurements? Chem. Phys. Lett. 200, 199-204 (1992).

51. Zander, C. et al. Detection and characterization of single molecules in aqueous solution. Appl. Phys. B 63, 517-523 (1996).

52. Maus, M. et al. An experimental comparison of the maximum likelihood estimation and nonlinear least-squares fluorescence lifetime analysis of single molecules. Anal. Chem. 73, 2078-2086 (2001).

53. Nettels, D., Gopich, I. V., Hoffmann, A. A. \& Schuler, B. Ultrafast dynamics of protein collapse from single-molecule photon statistics. Proc. Natl Acad. Sci. 104, 2655-2660 (2007)

54. Chung, H. S., McHale, K., Louis, J. M. \& Eaton, W. A. Single-molecule fluorescence experiments determine protein folding transition path times. Science. 335, 981-984 (2012).

55. Keller, B. G., Kobitski, A., Jäschke, A., Nienhaus, U. G. \& Noé, F. Complex RNA folding kinetics revealed by single-molecule FRET and hidden markov models. J. Am. Chem. Soc. 136, 4534-4543 (2014).

56. Pirchi, M. et al. Photon-by-photon hidden markov model analysis for microsecond single-molecule FRET kinetics. J. Phys. Chem. B 120, 13065-13075 (2016).

57. Gopich, I. V. \& Szabo, A. Theory of the energy transfer efficiency and fluorescence lifetime distribution in single-molecule FRET. Proc. Natl Acad. Sci. 109, 7747-7752 (2012).

58. Ingargiola, A., Weiss, S. \& Lerner, E. Monte carlo diffusion-enhanced photon inference: distance distributions and conformational dynamics in singlemolecule FRET. J. Phys. Chem. B 122, 11598-11615 (2018).

59. Gopich, I. V. \& Szabo, A. Decoding the pattern of photon colors in singlemolecule FRET. J. Phys. Chem. B 113, 10965-10973 (2009).

60. Müller, B. K., Zaychikov, E., Bräuchle, C. \& Lamb, D. C. Pulsed interleaved excitation. Biophysical J. 89, 3508-3522 (2005).

61. Laurence, T. A., Kong, X., Jager, M. \& Weiss, S. Probing structural heterogeneities and fluctuations of nucleic acids and denatured proteins. Proc. Natl Acad. Sci. 102, 17348-17353 (2005).

62. Hohng, S., Joo, C. \& Ha, T. Single-molecule three-color FRET. Biophysical J. 87, 1328-1337 (2004).

63. Clamme, J.-P. \& Deniz, A. A. Three-color single-molecule fluorescence resonance energy transfer. ChemPhysChem 6, 74-77 (2005).

64. Lee, N. K., Koh, H. R. \& Kim, S. K. Folding of 8-17 deoxyribozyme studied by three-color alternating-laser excitation of single molecules. J. Am. Chem. Soc. 129, 15526-15534 (2007)

65. Lee, N. K. et al. Three-color alternating-laser excitation of single molecules: monitoring multiple interactions and distances. Biophysical J. 92, 303-312 (2007).

66. Lee, S., Lee, J. \& Hohng, S. Single-molecule three-color FRET with both negligible spectral overlap and long observation time. PLoS One 5, e12270 (2010).

67. Stein, I. H., Steinhauser, C. \& Tinnefeld, P. Single-molecule four-color FRET visualizes energy-transfer paths on DNA origami. J. Am. Chem. Soc. 133, 719-726 (2011).

68. Yim, S. W. et al. Four-color alternating-laser excitation single-molecule fluorescence spectroscopy for next-generation biodetection assays. Clin. Chem. 58, 707-716 (2012).
69. Ratzke, C., Hellenkamp, B. \& Hugel, T. Four-colour FRET reveals directionality in the Hsp90 multicomponent machinery. Nat. Commun. 5, 4192 (2014).

70. Tsukanov, R., Tomov, T. E., Berger, Y., Liber, M. \& Nir, E. Conformational dynamics of DNA hairpins at millisecond resolution obtained from analysis of single-molecule FRET histograms. J. Phys. Chem. B 117, 16105-16109 (2013).

71. Peter, M. F. et al. Studying conformational changes of the yersinia Type-IIIsecretion effector YopO in solution by integrative structural biology. Structure 27, 1416-1426 (2019).

72. Harris, P. D. et al. Multi-parameter photon-by-photon hidden markov modeling dataset. https://zenodo.org/record/5902313 (2021).

73. Harris, P. D., Hamdan, S. M. \& Habuchi, S. Relative contributions of base stacking and electrostatic repulsion on DNA nicks and gaps. J. Phys. Chem. B 124, 10663-10672 (2020).

74. Biernacki, C., Celeux, G. \& Govaert, G. Assessing a mixture model for clustering with the integrated completed likelihood. IEEE Trans. Pattern Anal. Mach. Intell. 22, 719-725 (2000).

75. Celeux, G. \& Durand, J.-B. Selecting hidden Markov model state number with cross-validated likelihood. Computational Stat. 23, 541-564 (2008).

76. Harris, P. D. H2MMpythonlib: simulated models (2021). https://zenodo.org/ record/5535302.

77. Mächtel, R., Narducci, A., Griffith, D. A., Cordes, T. \& Orelle, C. An integrated transport mechanism of the maltose ABC importer. Res. Microbiol. 170, 321-337 (2019).

78. Kim, E. et al. A single-molecule dissection of ligand binding to a protein with intrinsic dynamics. Nat. Chem. Biol. 9, 313-318 (2013).

79. Ingargiola, A. Applying corrections in single-molecule FRET. bioRxiv083287 (2017). https://www.biorxiv.org/content/early/2017/02/01/083287.

80. Zickus, V. et al. Fluorescence lifetime imaging with a megapixel SPAD camera and neural network lifetime estimation. Sci. Rep. 10, 20986 (2020).

81. Harris, P. D. Fretbursts development version (2021). https://github.com/ harripd/FRETBursts/tree/polarization.

82. Cao, A.-M. et al. Allosteric modulators enhance agonist efficacy by increasing the residence time of a GPCR in the active state. Nat. Commun. 12, 5426 (2021).

83. Harris, P. D. H2mm tutorial (2021). https://doi.org/10.5281/zenodo.5566886

84. Peter, M. F. et al. Cross-validation of distance measurements in proteins by PELDOR/DEER and single-molecule FRET. bioRxiv 2020.11.23.394080 (2020). http://biorxiv.org/content/early/2020/11/23/2020.11.23.394080.abstract.

85. Ingargiola, A., Laurence, T., Boutelle, R., Weiss, S. \& Michalet, X. PhotonHDF5. Biophysical J. 110, 25-33 (2016).

86. Gebhardt, C. et al. Molecular and spectroscopic characterization of green and red cyanine fluorophores from the alexa fluor and AF series. ChemPhysChem 22, 1566-1583 (2021).

87. Ingargiola, A. et al. Multispot single-molecule FRET: High-throughput analysis of freely diffusing molecules. PLoS One 12, e0175766 (2017).

88. Ingargiola, A., Lerner, E., Chung, S. Y., Weiss, S. \& Michalet, X. FRETBursts: An open source toolkit for analysis of freely-diffusing Single-molecule FRET. PLoS ONE 11, 1-27 (2016).

89. Ingargiola, A. FOpenSMFS/PyBroMo: Version 0.8.1. zenodo.org (2019).

90. Hagai, D. \& Lerner, E. Systematic assessment of burst impurity in confocalbased single-molecule fluorescence detection using Brownian motion simulations. In: Molecules24 (2019) ISSN: 14203049. https://doi.org/10.3390/ molecules24142557.

\section{Acknowledgements}

We thank Gregor Hagelücken and Martin Peter from the Institute of Structural Biology (University of Bonn, GER) for providing YopO. We would like to thank Robert Quast and Emmanuel Margeat for insightful discussions regarding the implementation of $\mathrm{mpH}^{2} \mathrm{MM}$ for the analysis of 4-detector nsALEX measurements (2-color smFRET, with fluorescence anisotropies), based on their existing data ${ }^{82}$. We would also like to thank Demain Lieberman for his helpful discussion regarding implementation of $\mathrm{H}^{2} \mathrm{MM}$ code, and Bill Harris for his help in enabling the H2MM_C code to work on Windows and Linux. This paper was supported by the National Institutes of Health (NIH, grant R01 GM130942 to S.W. and E.L. as a subaward), the National Science Foundation (NSF, grants 1818147 and 1842951 to S.W.), the Human Frontiers Science Program (HFSP, grant RGP0061/2019 to S.W.), the Israel Science Foundation (ISF, grant 3565/20 to E.L., within the KillCorona - Curbing Coronavirus Research Program), the Milner Fund (to E.L.), and the Hebrew University of Jerusalem (start-up funds to E.L.). Work in the lab of T.C. was financed by Deutsche Forschungsgemeinschaft (SFB863, project A13 and GRK2062, project C03), an ERC Starting Grant (No. 638536 - SM-IMPORT to T.C.) and by the Center of Nanoscience Munich (CeNS).

\section{Author contributions}

E.L. performed HP3 nsALEX measurements. C.G. performed MalE nsALEX measurements. A.N. and T.C. performed YopO $\mu$ sALEX experiments. P.D.H. analyzed data and 
contributed analytical tools. P.D.H. \& E.L. designed and performed the research and composed the initial manuscript. P.D.H., A.N., C.G., T.C., S.W. \& E.L. discussed the data and contributed to the final version of the manuscript.

\section{Competing interests}

The authors declare no competing interests.

\section{Additional information}

Supplementary information The online version contains supplementary material available at https://doi.org/10.1038/s41467-022-28632-x.

Correspondence and requests for materials should be addressed to Paul David Harris or Eitan Lerner.

Peer review information Nature Communications thanks the anonymous reviewers for their contribution to the peer review of this work. Peer reviewer reports are available.

Reprints and permission information is available at http://www.nature.com/reprints
Publisher's note Springer Nature remains neutral with regard to jurisdictional claims in published maps and institutional affiliations.

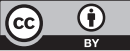

Open Access This article is licensed under a Creative Commons Attribution 4.0 International License, which permits use, sharing, adaptation, distribution and reproduction in any medium or format, as long as you give appropriate credit to the original author(s) and the source, provide a link to the Creative Commons license, and indicate if changes were made. The images or other third party material in this article are included in the article's Creative Commons license, unless indicated otherwise in a credit line to the material. If material is not included in the article's Creative Commons license and your intended use is not permitted by statutory regulation or exceeds the permitted use, you will need to obtain permission directly from the copyright holder. To view a copy of this license, visit http://creativecommons.org/ licenses/by/4.0/.

(C) The Author(s) 2022 